\title{
EFICIENCIA TÉCNICA, EFICIENCIA ECONÓMICA Y EFICIENCIA DINÁMICA
}

\author{
IVÁN CACHANOSKY*
}

Resumen: ¿Cómo determinar qué y cuánto producir? Esta es una de las grandes incógnitas de la economía. El presente trabajo propone estudiar las recetas ofrecidas por la economía convencional, el keynesianismo y la Escuela Austriaca de Economía poniendo en tela de juicio el uso de los supuestos y de las herramientas matemáticas. Además, se analizará el concepto de Eficiencia Dinámica, el cual brinda un aporte al debate. La importancia de analizar las distintas recetas se centra en resaltar que el uso erróneo de supuestos pueda llevar a conclusiones equivocadas por muy lógicas que parezcan.

Palabras clave: Eficiencia Técnica, Eficiencia Económica, Eficiencia Dinámica, Frontera de Posibilidades de Producción, Curvas de Indiferencia.

Clasificación JEL: B10, B25, B41, C61, D24, D50, O12, O33.

Abstract: How to determine how much and what to produce? This is one of the great unknowns in economics. This paper proposes to study the recipes offered by the conventional economics, the keynesian Economics, and the Austrian school of economics, questioning the use of assumptions and the mathematical tools. In addition, we will analyze the concept of dynamic efficiency, which provides a contribution to the debate. The importance of analyzing the various recipes focuses on highlight that the misuse of assumptions may lead to erroneous conclusions by very logical they seem.

Key words: Technical Efficiency, Economic Efficiency, Dynamic Efficiency, Production Possibilities Frontier, Indifference Curves.

JEL Classification: B10, B25, B41, C61, D24, D50, O12, O33.

* Licenciado en Administración de Empresas y candidato a Magister en Economía Aplicada de la Universidad Católica Argentina. Es investigador de la Fundación Libertad y Progreso. Correo electrónico: ivan.cachanosky@libertadyprogreso.org / ivan@corporatet.com. 
El presente trabajo pretende mostrar visiones opuestas acerca de la asignación de recursos y su eficiencia. La importancia del tema a estudiar es que en la actualidad los diversos manuales de economía presentan una sola postura del tema, el de la economía convencional, sin mencionar las críticas a los modelos económicos. En dichos manuales se observa que existen dos tipos de eficiencia, la eficiencia técnica y la eficiencia económica. La primera se encuentra relacionada con el uso de la capacidad instalada mientras que la segunda cuestiona si los recursos utilizados se encuentran asignados de manera eficiente. La pregunta clave, una vez diferenciados ambos tipos de eficiencia, es: ¿cómo llegar a la eficiencia económica? A esta pregunta responden diferentes escuelas.

Para intentar demostrar la importancia del análisis, el trabajo se basará en los siguientes 5 puntos:

1. La eficiencia técnica. Este punto se enfocará en explicar el concepto de eficiencia técnica y en mostrar por qué no es suficiente para guiar a la economía.

2. La eficiencia económica. En esta sección se verá la importancia de la misma para poder satisfacer las necesidades de los consumidores y además se planteará el dilema de si ésta es alcanzable o no. Se observará, en primera instancia, el enfoque de la economía convencional y luego se verán críticas a dicho enfoque.

3. La eficiencia técnica y Keynes. Este tercer punto se centrará en mostrar por qué las políticas de tinte keynesiano jamás podrán alcanzar la eficiencia económica.

4. La solución de la Escuela Austriaca de Economía. El presente apartado propondrá una solución alternativa a la de la economía convencional más completa y con un enfoque más enriquecedor.

5. La eficiencia dinámica. Por último, este punto se encuentra dirigido a brindar una visión más innovadora y actualizada del asunto.

El objetivo es estudiar qué mecanismo es más correcto para lograr la eficiencia económica, en caso de que ésta pueda lograrse, y si el concepto de eficiencia dinámica suple o se complementa con el de eficiencia económica. Es de importancia destacar que tanto 
la postura presentada por la economía convencional como la de la Escuela Austriaca tienen sus puntos fuertes dependiendo de si se aceptan o rechazan ciertas premisas. De todos modos, el presente trabajo intentará demostrar por qué el enfoque de la Escuela Austriaca de Economía es mucho más sólido y consistente que el de la economía convencional.

\section{I \\ EFICIENCIA TÉCNICA}

La eficiencia técnica refleja si los recursos son explotados al máximo de su capacidad productiva o no. Es decir, si hay capacidad ociosa de los factores productivos o si están siendo usados al cien por ciento. En economía, hay un famoso gráfico llamado Frontera de Posibilidades de Producción (FPP) que resume esta idea. El gráfico es el siguiente:

GRÁFICO 1

FRONTERA DE POSIBILIDADES DE PRODUCCIÓN

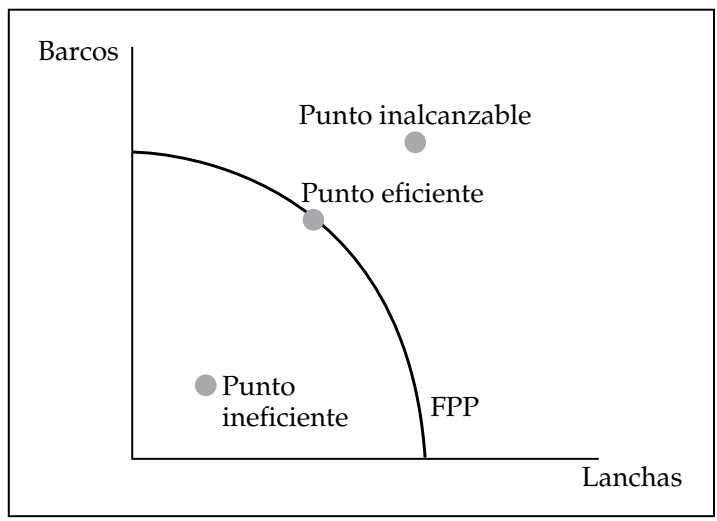

En el gráfico se puede observar que se analizan la producción de dos bienes, la producción de «Lanchas» en el eje de las X y la producción de «Barcos» en el eje de las Y. La Frontera de Posibilidades de Producción es la curva cóncava representada por FPP. 
Un punto por debajo de la FPP quiere decir que no se están utilizando todos los recursos productivos, es decir, hay capacidad ociosa alcanzando un punto ineficiente. Un punto sobre la curva FPP implicaría que se están utilizando todos los recursos disponibles, es decir, no hay capacidad ociosa y se alcanza un punto tecnológicamente eficiente. Por último, a un punto por arriba de la FPP se lo llama inalcanzable debido a que no se poseen los suficientes recursos para alcanzar dicho punto. En otras palabras, estando por debajo de la curva FPP, se podrían estar produciendo más lanchas y/o más barcos pero no se está llevando a cabo dicha producción y sobran recursos. Situados sobre la curva FPP ya no hay recursos para producir más barcos ni lanchas y un punto por encima de la curva FPP refleja una situación inalcanzable porque no existen los recursos para producir dichas cantidades de barcos y lanchas. Para poder llegar al punto inalcanzable se requiere de un avance tecnológico que desplace la FPP hacia la derecha, por ejemplo:

GRÁFICO 2

EXPANSIÓN DE LA FRONTERA

DE POSIBILIDADES DE PRODUCCIÓN

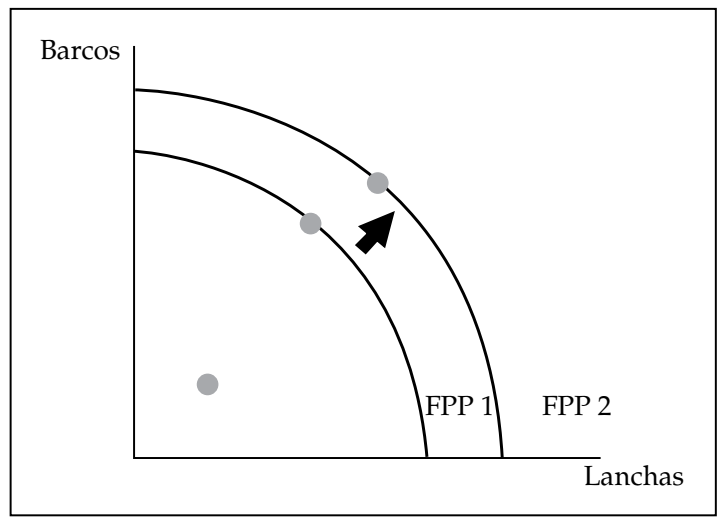

Los avances tecnológicos, la utilización de mejores bienes de capital, el know how, permiten producir una mayor cantidad de bienes y servicios y desplazan la FPP hacia la derecha generando un 
desarrollo económico. Este punto se verá más adelante con mayor detalle. Por el momento, lo importante es focalizarse en el gráfico anterior donde todavía no se presencian avances tecnológicos.

El hecho de estar sobre la FPP es lo que se denomina eficiencia técnica. Se están aprovechando todos los recursos disponibles, técnicamente se es eficiente para producir un bien determinado. Por ejemplo, supongamos que dichos factores productivos son utilizados sobre la FPP en el punto señalado del gráfico. Esto nos permite explotar al máximo nuestros recursos y así podremos producir, por ejemplo, 8 barcos y 6 lanchas. ${ }^{1}$ Pero para la eficiencia económica lo que es importante analizar es: ¿en qué cantidades los consumidores están demandando barcos y lanchas? ¿Más barcos que lanchas, a la inversa o equilibrado? Veamos un ejemplo. Supongamos que otro productor sostiene que se demandan más lanchas que barcos. Se representará nuevamente el gráfico agregando un triángulo sobre la FPP, el cual refleja que se estarían destinando los recursos a producir más cantidad de lanchas y menos de barcos.

GRÁFICO 3

EFICIENCIA TÉCNICA EN LA FRONTERA

DE POSIBILIDADES DE PRODUCCIÓN

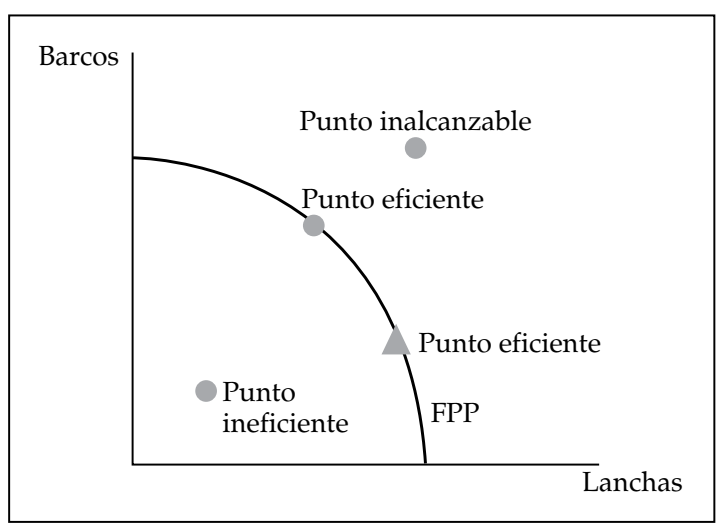

${ }^{1}$ Si bien en el gráfico de la FPP, el bien X y el bien $\mathrm{Y}$ representan una economía con dos bienes y puede resultar más sencillo ver como destinar los recursos, en el mundo real, al haber una gran cantidad de bienes y servicios resulta más difícil poder determinar qué bienes se encuentran demandando en ese momento los consumidores. 
¿Cómo saber qué punto sobre la FPP es más eficiente? ¿El círculo o el triángulo? ¿U otro que no se haya marcado? ¿En qué cantidades se encuentran los consumidores demandando los bienes que ofrece el mercado? Ésa es la pregunta que responderá la eficiencia económica.

Hasta aquí, lo que podemos afirmar con certeza es que tanto en el círculo como en el triángulo, puntos ubicados sobre la FPP, se están utilizando todos los recursos productivos y no hay capacidad ociosa. En el caso del círculo se producen más barcos que lanchas, en el caso del triángulo se producen más lanchas que barcos pero, nuevamente, ¿qué están demandando las personas? Como se dijo, esta pregunta no puede ser respondida por la eficiencia técnica. Como ya se mencionó, dicha eficiencia sólo nos muestra si se están utilizando los factores de producción en su totalidad, pero no si se están utilizando en producir los bienes que la gente demanda.

Por último, cabe mencionar que existen diversas formas de la FPP dependiendo de su pendiente. Es lo que se llama Relación de Transformación del Producto (RTP) que en palabras del economista Walter Nicholson se define como «la relación de transformación del producto entre dos productos es la pendiente con signo negativo de la frontera de posibilidades de producción de estos productos». ${ }^{2}$ En otras palabras, cuanto mayor sea la pendiente negativa más cantidades del eje de las $Y$ (barcos) habrá que renunciar para obtener una unidad adicional de X (lanchas); es lo que se llama una RTP creciente. De todas maneras, la magnitud de la pendiente negativa de la FPP no afecta las conclusiones ni el enfoque del presente trabajo.

II

EFICIENCIA ECONÓMICA

Se ha visto que estar en un punto sobre la FPP nos garantiza la eficiencia técnica, es decir, no poseer capacidad ociosa. Por ejemplo, estamos utilizando los factores productivos para producir

${ }^{2}$ Nicholson, W. (2005), p. 432. 
1000 teléfonos. Sin embargo, lo realmente importante a analizar es si la gente se encuentra demandando teléfonos. En una economía que ofrezca una diversa cantidad de bienes y servicios no solo se posee el problema de asignar eficientemente los recursos, sino que además se debe estudiar si se están utilizando para los bienes que las personas demandan. El problema que posee la economía es resolver el dilema de asignación.

Aquí es donde entra en juego la eficiencia económica. Aquel punto, sobre la FPP, que a la vez sea utilizado para producir los bienes que demandan los consumidores alcanzará la eficiencia económica. De lo contrario solo estamos ante la presencia de eficiencia técnica produciendo bienes que pueden no ser demandados, o bien, que no son demandados en esas cantidades mal asignando los recursos. Sintetizando, se utilizan la totalidad de los recursos sin tener capacidad ociosa pero para producir bienes que no serán comprados en su totalidad por los consumidores.

Si se logra encontrar el punto sobre la FPP que permita producir los bienes que los consumidores demandan entonces habremos logrado la eficiencia económica. Puede ocurrir que consumidores demanden barcos y lanchas pero no en las cantidades que se están produciendo. En ese caso, si se alcanzaría la eficiencia técnica pero no la eficiencia económica.

¿Cómo alcanzar la eficiencia económica? La respuesta dependerá de la escuela que se estudie. Se examinará primero la respuesta que brinda la economía convencional.

\section{La eficiencia económica en la economía convencional}

Para entender la lógica que planteará la economía convencional es importante primero explicar y desarrollar el concepto de curva de indiferencia. ${ }^{3}$ Una curva de indiferencia muestra la relación entre dos bienes en donde el consumidor se mantiene indiferente. Es decir, siempre que el consumidor se encuentre sobre la curva de

${ }^{3}$ Esto es importante debido a que la curva de indiferencia forma parte de la explicación de cómo lograr la eficiencia económica. No podría ser explicada con la ausencia del concepto de curva de indiferencia. 
indiferencia le será indistinto consumir más bienes de $\mathrm{X}$ y menos de $\mathrm{Y}$ o viceversa. El siguiente gráfico puede resultar esclarecedor:

GRÁFICO 4

CURVAS DE INDIFERENCIA

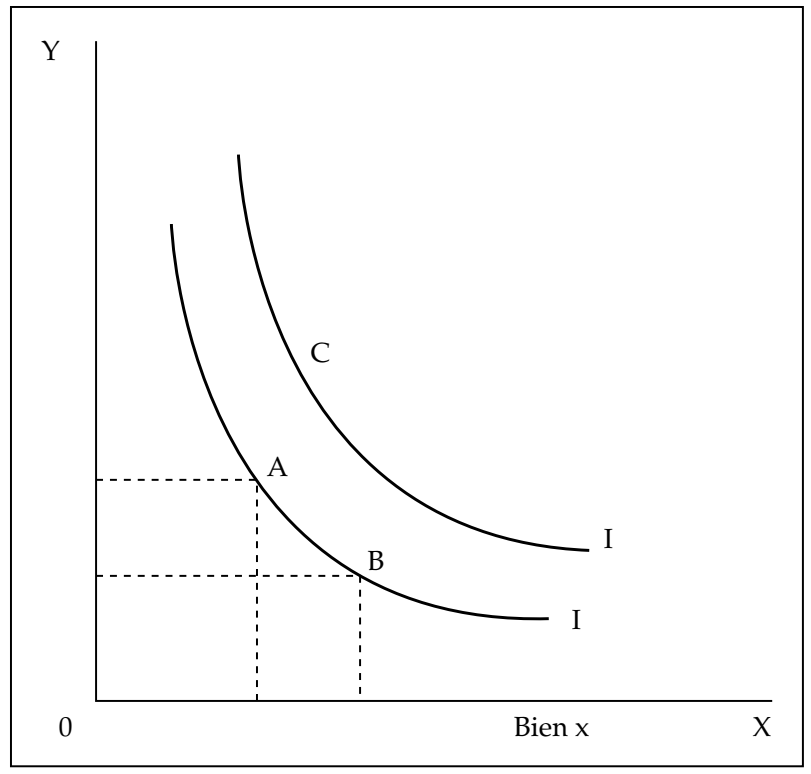

La curva de indiferencia I muestra distintas combinaciones de consumo para una persona en el cual la satisfacción total es la misma, o sea, cualquier punto de la curva le es indiferente. En el punto A consume más bienes de "Y» y menos de "X»; en el punto $B$ consume más bienes de « $X » y$ menos de « $Y »$. Lo importante es que le es indiferente estar en A o en B. En definitiva, la curva de indiferencia son diferentes combinaciones de consumo para el cual la persona se mantiene indiferente porque su nivel de satisfacción es el mismo. Tanto el punto A como el punto B le brindan el mismo nivel de utilidad al consumidor.

Cuanto más alejada esté la curva de indiferencia, mejor es la situación del consumidor porque significa que éste puede obtener más de ambos bienes. La curva de indiferencia I brinda menor utilidad que la curva de indiferencia $\mathrm{I}^{\prime}$. 
Una vez comprendido el concepto de curva de indiferencia se está en condiciones de ver la solución que sugiere la economía convencional para lograr la eficiencia económica. Esto sucede en el punto en el cual la curva de indiferencia se hace tangente a la FPP. Gráficamente sería de la siguiente manera:

GRÁFICO 5

FRONTERA DE POSIBILIDADES DE PRODUCCIÓN Y CURVA DE INDIFERENCIA

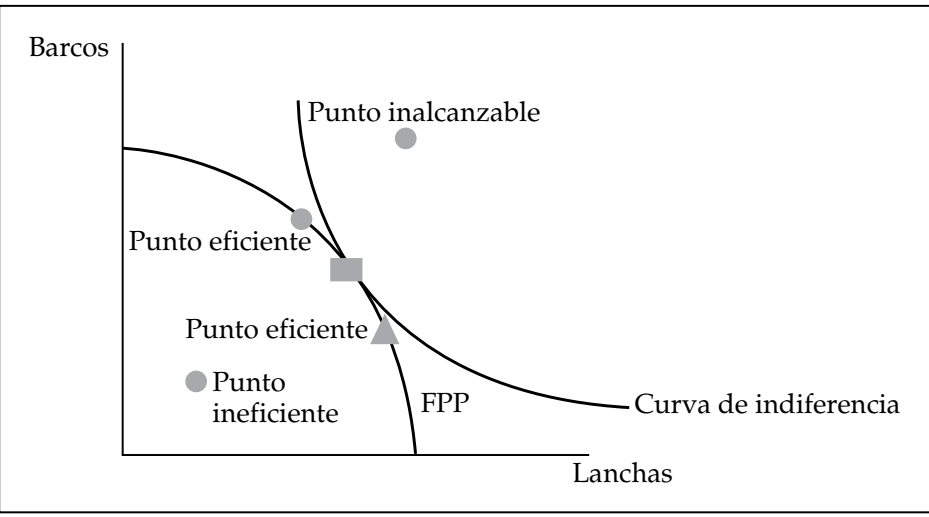

Podemos observar en el gráfico, que el cuadrado muestra el punto en donde la FPP y la curva de indiferencia son tangentes. En ese punto se igualan una situación de eficiencia técnica que además es demandada por los consumidores. Por lo tanto, ese y sólo ese punto de la FPP representa la eficiencia económica. La gente se encuentra demandando cierta cantidad de barcos y de lanchas (el cuadrado negro en el gráfico) y además se están utilizando todos los recursos produciendo sobre la FPP. Cualquier otro punto sobre la FPP no logra la eficiencia económica, solo logra la eficiencia técni$c a$. Es importante volver a destacar que lo importante es lograr la eficiencia económica ya que de nada nos sirve ser eficientes para producir barcos y lanchas si después no serán demandados. Simplemente estaríamos desperdiciando recursos, destruyendo capital.

Si se produce un avance tecnológico y la FPP se desplaza hacia la derecha, la nueva FPP se encontrará con una mayor curva de indiferencia brindando un nuevo punto de eficiencia económica. 
Si se pusiera un freno aquí pareciera estar el problema resuelto. Para lograr eficiencia económica hay que encontrar el punto en donde la FPP sea tangente con la curva de indiferencia de los consumidores. Sin embargo, la curva de indiferencia posee algunos aspectos que complican el razonamiento planteado. Los autores de la Escuela Austriaca de Economía ${ }^{4}$ promueven un recalcado énfasis en la subjetividad del valor.

Carl Menger, Stanley Jevons y León Walras fueron los tres economistas que provocaron la revolución marginal derivando en el concepto del valor subjetivo. Este punto será de gran importancia dentro del pensamiento de la Escuela Austriaca de Economía. Al ser el valor subjetivo, sólo el individuo mismo sabe qué es lo que demanda. Es prácticamente imposible que un gobernante de turno pueda «leer» las necesidades de una población. ${ }^{5}$ De ahí la inviabilidad del socialismo y el intervencionismo, ya que carecerán de medios para saber qué es lo que las personas demandan ni con qué fines. La economía convencional pareciera ignorar la importancia de la subjetividad del valor al estudiar las curvas de indiferencia ya que asume que los bienes que se analizan son sustitutos. Para refutar este punto se analizará la validez de la curva de indiferencia desde dos perspectivas:

a) La curva de indiferencia del individuo y la curva de indiferencia de una sociedad.

b) El rol de las expectativas en las curvas de indiferencia.

También, se analizará el rol que juega la información para predecir en economía:

c) Información Perfecta.

\footnotetext{
${ }^{4}$ Carl Menger, Eugen Böhm Bawerk, Ludwig von Mises, Friedrich Hayek, Murray Rothbard y muchos otros.

5 A mayor tamaño de la población, menor será la probabilidad de una economía planificada. Es una relación inversa.
} 


\section{a) La curva de indiferencia del individuo}

y la curva de indiferencia de una sociedad

Para el caso planteado, barcos y lanchas serían sustitutos. Asumir el supuesto de que ambos bienes son sustitutos para una sociedad es errado ya que solo se puede dibujar la curva de indiferencia de personas particulares por separado. El economista Murray Rothbard hizo mucho énfasis en este punto. Casualmente los bienes que se tomaron como ejemplo (barcos y lanchas) son coincidentes con los que utilizó Rothbard en su libro Man, Economy and State. El punto que quiere remarcar Rothbard es que una persona A puede estar demandando un barco porque le gusta navegar, pero tal vez otra persona B lo demande como lugar de recreo para despejarse. Para el primer caso la lancha si representa un sustituto, pero para la persona B tal vez no; en cambio, una casa de recreo si puede ser un sustituto para la persona B. En palabras de Rothbard:

If the price of yachts becomes too high, the consumer can substitute expenditure on mansions, or he can substitute books for television sets, etc. $^{6}$

Si bien Rothbard utiliza el ejemplo analizando sustitutos de un posible monopolio, el ejemplo no deja de ser aplicable para el presente trabajo. En síntesis, le corresponde solamente al consumidor evaluar que bienes considera él que son sustitutos y complementarios. No es tarea del economista, ni del político, ni del empresario. Jamás podrían hacerlo debido a la subjetividad del valor. Una persona A puede desayunar café porqué la cafeína hace que se despierte, una persona B puede desayunar café porque se siente bien yendo a trabajar con algo en el estómago. Para la persona A puede que el té no sea un sustituto ya que si el té no logra despertarlo no puede ser sustituto del café y dicha persona tomaba café en primera instancia porque lo que demandaba era despertarse. En cambio para le persona B sí puede ser un sustituto el té ya que demandaba ir a trabajar con algo en el estómago. Una

6 Rothbard, Murray N. (1962), p. 596. 
vez más, se puede observar que depende del individuo evaluar dichas cuestiones. Sin embargo, cualquier manual de microeconomía decretaría que el té y el café son sustitutos. ${ }^{7}$

Yendo a nuestro caso concreto, podemos concluir que no se puede afirmar que los barcos y los yates sean sustitutos para toda una sociedad. Sí puede dibujarse la curva de indiferencia de un individuo, pero de allí a asumir que esa curva de indiferencia puede ser cierta para una sociedad, como lo hacen los manuales con el café y el té por ejemplo, es un error. Los bienes que se producen son demandados por muchas personas, el no poder representar gráficamente una curva de indiferencia para el conjunto de la sociedad imposibilita a la economía convencional encontrar el punto de tangencia entre la FPP y la curva de indiferencia. Al no poder definir si los bienes son sustitutos o complementarios para la sociedad, no es posible dibujar las curvas de indiferencia. Este es un primer problema que presenta la solución brindada por la economía convencional: es posible dibujar una curva de indiferencia para un individuo pero no para la sociedad. De esta manera se hace evidente el problema de no poder graficar la tangencia de la FPP con la curva de indiferencia y nuevamente la imposibilidad de no poder determinar la eficiencia económica. Se vuelva al punto inicial.

\section{b) El rol de las expectativas en la curva de indiferencia}

Sin embargo, éste no es el único problema que puede presentar una curva de indiferencia. Existe un segundo problema y es que dicha curva no tiene en cuenta el rol de las expectativas. Oskar Morgenstern en su paper «Thirteen Critical Points in Contemporany Economic Theroy: An Interpretation» señala este problema de la curva de indiferencia. El autor sostiene que dentro de una misma curva de indiferencia puede existir un punto preferible a otro, ${ }^{8}$

${ }^{7}$ Lo cual puede ser cierto para algunas personas. Pero no puede decretarse que es cierto para el conjunto de la sociedad.

8 Este punto tiene validez sólo en el caso que se tenga en cuenta el rol de las expectativas futuras. 
es decir, el consumidor no estaría siendo indiferente dentro de la misma curva de indiferencia. A continuación se mostrará el gráfico que el autor posee en su trabajo.

GRÁFICO 6

ANÁLISIS CURVA DE INDIFERENCIA

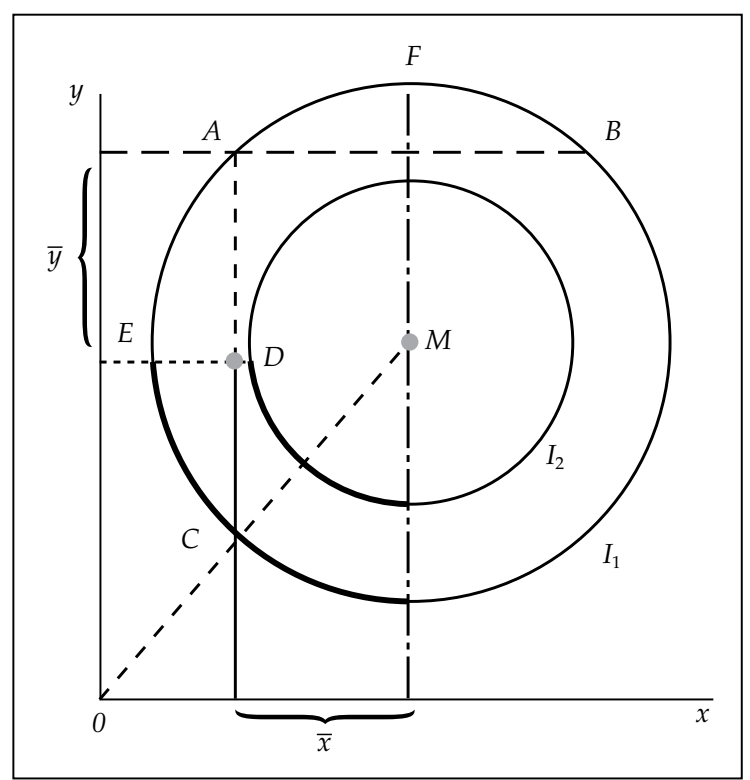

Fuente: Morgenstern, O. Thirteen Critical Points in Contemporary Economic Theory: An Interpretation. 1972.

Para comprender el gráfico de Morgenstern es mejor citar sus propias palabras:

Consider the classical diagram, Pareto s «colline de plaisir», with $\mathrm{M}$ the highest point. $\mathrm{A}$ and $\mathrm{B}$ are on $\mathrm{I}_{1}$, defined as indifferent; furthermore $\mathrm{M}>\mathrm{I}_{\mathrm{n}}>\ldots>\mathrm{I}_{2}>\mathrm{I}_{1} . \mathrm{A}=\mathrm{B}$ is the case only if the individual is unable, or not allowed, to throw away those excess amounts of both $\mathrm{x}$ and $\mathrm{y}$ which would bring him to $\mathrm{M}$, his maximum of utility. Occasionally in the literature «free disposal» is mentioned, but no further analysis given... ${ }^{9}$

9 Morgenstern, O. (1972), p. 1179. 
Pero para entenderlo mejor, es importante concentrarse en las partes de las curvas de indiferencia que se encuentran en un negro más resaltado. Se puede observar que el punto $\mathrm{E}$ y el punto $\mathrm{C}$ se encuentran sobre la misma curva de indiferencia. Morgenstern sostiene, que al entrar las expectativas en juego las condiciones puedan cambiar. Si se espera que el precio de $X$ disminuya, el consumidor probablemente se vea motivado a no consumir hoy para consumir más en el futuro. ${ }^{10}$ Suponiendo que una persona se encuentra en el punto E, al disminuir el precio de $X$ puede comprar más bienes de ese bien y así moverse a la curva de indiferencia $\mathrm{I}_{2}$. Si una persona se encuentra en el punto E se puede observar claramente que fácilmente se puede desplazar al punto $\mathrm{D}$ alcanzando una curva de indiferencia mayor. En cambio si una persona se encuentra en el punto $C$, el precio de $X$ puede bajar, la persona podrá comprar más bienes de $X$ pero no alcanzará la curva de indiferencia $\mathrm{I}_{2}$. En el gráfico se puede observar la diferencia entre el punto E y C. Bajo la lógica que plantea Morgenstern, con expectativas de que los precios de $\mathrm{X}$ van a disminuir, el punto E es preferible al C y sin embargo se encuentran sobre la misma curva de indiferencia. Al entrar en juego el rol de las expectativas se pone en tela de juicio que los puntos sobre la misma curva de indiferencia sean indiferentes. Además, cada individuo puede tener expectativas muy distintas.

Este error surge de aplicar matemática a la teoría económica. No hay que olvidar que en la ciencia económica, la causalidad es teleológica ${ }^{11}$ y que por lo tanto los pasajes de términos no son válidos. En economía las constantes cambian continuamente, es por eso que el uso de las matemáticas no siempre es apropiado.

Por otra parte, como señala Alberto Benegas Lynch (h), la indiferencia anularía la capacidad de acción humana para elegir. En sus propias palabras:

10 Cuando baje el precio de $X$.

11 Para causalidad teleológica se puede ver el siguiente párrafo de Mises en su tratado de Economía, La Acción Humana: «Si tomamos el término de causalidad en su sentido más amplio, la teleología puede considerarse como una rama del análisis causal. Las causas finales son las primeras de todas las causas. La causa de un hecho es siempre determinada acción o cuasi acción que apunta a un determinado objetivo.» 
... la falacia crucial de la «indiferencia» es que no tiene relación alguna con la acción. Si un hombre fuera realmente indiferente entre dos alternativas no podría elegir entre ellas y, por ende, no hay acción. Toda acción demuestra preferencia: preferencia de una posibilidad frente a otras. No hay entonces rol alguno para el concepto de la indiferencia en la economía. ${ }^{12}$

\section{c) Información Perfecta}

El problema de decidir cuál es la mejor manera de utilizar el conocimiento que inicialmente se encuentra disperso entre toda la gente es, cuando menos, uno de los principales problemas de la política económica. ${ }^{13}$

Esta cita de Hayek resume de manera clara el problema desde el punto de vista de la Escuela Austriaca de Economía. El otro gran problema que tiene la economía convencional en la utilización de sus manuales es el supuesto de que todos los individuos poseen información completa. Si esto fuera así, sería cierto que lo único que resta hacer es ordenar los datos ya disponibles. Es así como se llega a problemas de maximización y minimización. Debido a que los datos se encuentran dados solo queda «acomodarlos». Sin embargo, no es así como funciona el mundo real. Lo que Hayek plantea es que la economía convencional está salteando un paso, primero hay que descubrir los datos y luego maximizarlos. ${ }^{14}$ Hayek distingue en dos tipos de conocimiento: a) El conocimiento científico y b) conocimiento no científico.

El primero es aquel que podría llegar a ser dominado por los expertos y científicos. Pero, como señala Hayek, se suele creer que es el único tipo de conocimiento cuando no es así. Existe también el conocimiento no científico que es desorganizado y este conocimiento depende de circunstancias de tiempo y de lugar. Este segundo tipo de conocimiento es el que los científicos no pueden dominar y depende de cada individuo. Si el gobernante de turno

\footnotetext{
12 Benegas Lynch (h) (2011), p. 75.

13 Hayek (1945), p. 159.

14 Este punto se discutirá en más detalle en la sección siguiente.
} 
logra triunfar en la difícil tarea de ordenar el conocimiento científico aún le restaría ordenar el conocimiento no científico y eso le resultara imposible porque como se mencionó; éste depende y varía según cada individuo y según el tiempo y el lugar y por ende no puede ser comunicado bajo ninguna forma de estadística. De allí que Hayek señale que el planeamiento no puede ser centralizado, debe ser descentralizado. En sus propias palabras:

Si estamos de acuerdo en que el problema económico de la sociedad es principalmente un problema de adaptarse rápidamente a los cambios en las circunstancias específicas de tiempo y lugar, de ahí se sigue que las decisiones últimas deben dejarse a las personas que conocen estas circunstancias; que conocen directamente los cambios significativos y los recursos que están directamente a la mano para hacerles frente. ${ }^{15}$

El problema de la información se volverá a ver brevemente más adelante. Pero si la información no es perfecta y se encuentra dispersa como sostiene Hayek, entonces no poseemos los datos necesarios para lograr que la FPP toque tangencialmente las curvas de indiferencia de los consumidores.

En respuesta, la economía convencional sostiene que lo importante en los supuestos que se toman es su capacidad de predicción más allá de si son reales o no. Por otra parte, se le objeta a la economía convencional que predecir no es lo mismo que explicar y lo que la ciencia económica debe hacer es intentar explicar. ${ }^{16}$ En el presente trabajo se adoptará la postura crítica a la de Milton Friedman. Para quienes crean en ellas, las líneas que siguen las encontrarán refutables.

Una vez aclarado ese punto se puede proseguir con la idea de que si bien la economía convencional brinda una solución para llegar a la eficiencia económica, la teoría que ofrece sobre curvas de indiferencia recibe algunas críticas que invalidarían el resultado de alcanzar la eficiencia económica. Ello sería posible si el conocimiento

15 Hayek, Friedrich, op. cit., p. 163.

16 Para un análisis detallado del tema ver: von Mises, Ludwig. Ultimate Foundation of Economic Science. Indianapolis: Liberty Fund. 1962. Hayek, Friedrich The Counter-Revolution of Science. Indianapolis: Liberty Press. 1952. 
fuera perfecto, como sostiene la economía convencional. Pero dado que no lo es, no hay manera de ordenar matemáticamente los datos para lograr la maximización del problema. O en palabras de Kirzner:

... la decisión humana no se puede explicar puramente en términos de maximización, de una reacción «pasiva» que toma la forma de adoptar el «mejor» curso de acción, según lo marcan las circunstancias. ${ }^{17}$

Se debe tener en cuenta además, que si el conocimiento fuera perfecto estaríamos en el campo de la no acción porque todo se ajustaría automáticamente. La acción humana que menciona Mises se basa en la incertidumbre futura y por eso al referirse a los empresarios sostiene que se maneja con precios esperados. El asumir conocimiento perfecto se estaría suponiendo transitar el campo de la no acción. La siguiente cita de Alberto Benegas Lynch (h) puede resumir esta última idea planteada:

La acción humana implica incertidumbre puesto que si el hombre conociera el futuro no elegiría ni optaría, puesto que las cosas inexorablemente se sucederían según lo ya conocido de antemano (incluyendo por definición los movimientos del sujeto que posee ese conocimiento) lo cual significaría que no hay acción. ${ }^{18}$

Nuevamente reaparece el dilema sobre cómo llegar a la eficiencia económica. Aquella persona que no se encuentre convencida de la solución que brinda la economía convencional estaría volviendo al punto de inicio de este trabajo. La Escuela Austriaca de Economía plantea una alternativa para lograr una solución, pero antes de ver qué solución o alternativa plantea, se realizará un «paréntesis» para comentar la opinión de Keynes y la solución que propuso en la crisis del 30. Una vez analizado el punto de Keynes se pasará a plantear la alternativa de la Escuela Austriaca de Economía.

\footnotetext{
17 Kirzner (1990), p. 53.

18 Benegas Lynch (h) (1972), p. 66.
} 


\section{III LA EFICIENCIA TÉCNICA Y KEYNES}

$\mathrm{Al}$ aparecer la crisis del 30 el economista John Maynard Keynes tomó protagonismo tras sostener que el capitalismo es el responsable de la crisis ${ }^{19}$ y puso en tela de juicio la mano invisible de Adam Smith. El argumento de Keynes llevado a nuestro gráfico es: «Nos encontramos en un punto por debajo de la FPP. Dejemos que el gobierno intervenga para empujar el punto ineficiente a un punto sobre la FPP y así lograr una eficiencia técnica». En realidad, Keynes no habló propiamente de la eficiencia técnica sino que su propuesta era ir hacia el pleno empleo, lo que indirectamente implicaba trasladarse a un punto de la FPP ofreciendo trabajo a las personas. El problema, como ya se mencionó, es que la eficiencia técnica no nos garantiza la eficiencia económica, y si esto no se logra lo único que lograremos tarde o temprano es desperdiciar recursos. ${ }^{20}$

Para poder entender mejor el riesgo de la política emisora de Keynes se puede ver el siguiente ejemplo. Supongamos que la economía se encuentra en un punto por debajo de la FPP y Keynes sugiere empujar la economía hacia el pleno empleo, lo que implica intentar ir hacia un punto sobre la FPP para producir cierta cantidad de barcos y lanchas. Pasa el tiempo y el mercado da señales de que no se están demandando barcos y lanchas en esas cantidades. Hasta aquí logramos desperdiciar recursos y estos vuelven a estancarse y volvemos a ubicarnos en un punto por debajo de la FPP volviendo a obtener capacidad ociosa. Ahora supongamos que un keynesiano proponga volver al pleno empleo dirigiéndose hacia otro punto de la FPP ya que cree que se produjeron pocos barcos y muchas lanchas y la gente demandaba a la inversa. Si este punto es el que nos permite producir los bienes

19 Keynes (1930).

20 Es importante entender la época en que vivía Keynes. La crisis del 30 fue muy fuerte y esto lo llevó a que sus ideas y escritos se centren en salir de la crisis como objetivo primordial. No es lo mismo plantear «remedios» keynesianos ante la presencia de fuertes crisis que como política económica. Es decir, no es lo mismo Keynes que los keynesianos. 
que nos llevan a la eficiencia económica todo marchara bien, pero si el gobernante de turno erró en su predicción, nuevamente estaremos desplazándonos hacia un punto por debajo de la FPP. Es por eso que se dice que Keynes proponía «que una persona cabe un pozo y otro lo tape». ${ }^{21}$ Este mecanismo puede continuar y tal vez por iteración se llegue al punto de la eficiencia económica. Podría llegarse por iteración, pero las alternativas son tantas que se complica la probabilidad de acierto. El siguiente gráfico puede mostrar el problema:

\section{GRÁFICO 7}

\section{LA EFICIENCIA TÉCNICA Y KEYNES}

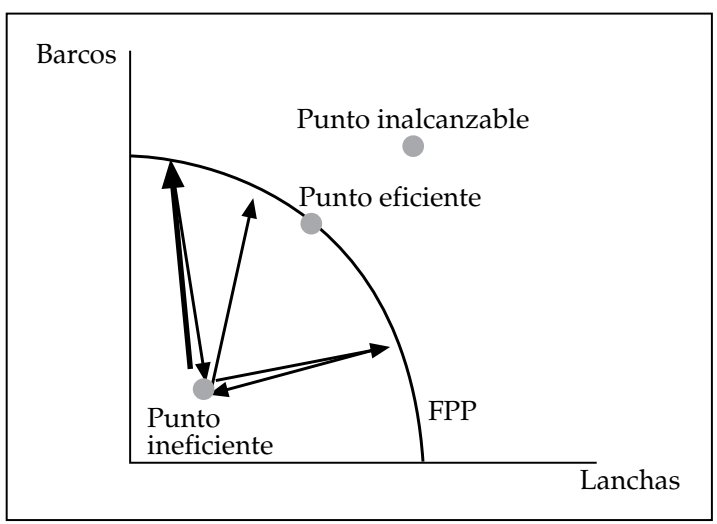

El recorrido comenzaría con la flecha más oscura. Keynes sostendría que hay que moverse hacia la $\mathrm{FPP}, 22$ pero al no lograr la eficiencia económica tarde o temprano termina moviéndose a un punto ineficiente nuevamente y volverá a incrementar el gasto para volver a llegar a un punto sobre la FPP, pero otra vez, si no es económicamente eficiente ocurrirá lo mismo y así sucesivamente. El problema sigue siendo que debido a la subjetividad del valor

${ }^{21}$ No son palabras de Keynes. Es su ejemplo llevado al extremo.

22 Siempre hablando implícitamente debido a que en realidad él mencionaba que hay que generar trabajo para acercarse al pleno empleo. 
el gobernante de turno no puede saber qué es lo que la gente está demandando.

Además como menciona Benegas Lynch (h) la inversión del estado no es una buena inversión porque sólo el libre mercado puede dirigir la asignación de recursos como se observará más adelante. Es una cuestión de costo de oportunidad:

Cuando el gobierno saca coactivamente recursos de la comunidad, su asignación técnicamente no puede llamarse «inversión», puesto que los sujetos actuantes en el mercado no estiman que dadas las circunstancias aquel específico consumo futuro traerá aparejado mayor rendimiento que la alternativa seleccionada por el mercado; de allí que, precisamente, se requiere la coerción. ${ }^{23}$

Otra opción es la del libre mercado elaborada por la Escuela Austriaca de Economía que se analizará a continuación.

IV

\section{LA SOLUCIÓN DE LA ESCUELA AUSTRIACA DE ECONOMÍA}

A grandes rasgos la Escuela Austriaca de Economía postula que: el valor es subjetivo; que se debe respetar el derecho de propiedad; que la información está dispersa, que los precios no deben intervenirse y que las nuevas oportunidades de beneficios son descubiertas por los empresarios. En mayor o menor medida éstas son las bases teóricas de la Escuela Austriaca de Economía. Se analizarán dichos puntos a continuación y se irá observando que esta escuela ve un enfoque muy distinto al del equilibrio general competitivo. Los autores de esta escuela no creen que los datos se encuentren dados y que simplemente haya que maximizarlos con ecuaciones matemáticas; por el contrario, ven a la economía como un proceso de aprendizaje con información dispersa donde el mercado tiende a guiar a los consumidores y productores hacia el equilibrio.

${ }^{23}$ Benegas Lynch (h), op. cit., pp. 368-369. 
Se comenzará por analizar la subjetividad del valor. Cada consumidor tiene su escala individual de valores, esto es lo que hace que el valor sea subjetivo. Una persona puede valorar un bien y otra persona puede valorar aún más el mismo bien (o puede valorarlo menos), o incluso no valorarlo en absoluto. Debido a que el valor es subjetivo es que puede existir el intercambio. Cuando dos personas realizan una transacción, la realizan porque esperan beneficiarse, de lo contrario no actuarían de esa manera. Gracias a que ambas personas valoran el mismo bien de manera distinta se produce el trato. Es lo que Mises denominó una «win-win situation». La subjetividad del valor tuvo sus orígenes en la revolución marginal con Carl Menger, Stanley Jevons y León Walras. Estos tres autores llegaron a la misma conclusión de manera simultánea. Fue dicha revolución la que solucionó el problema de la paradoja del valor. ¿Cómo era posible que un diamante que tiene poca utilidad tenga un valor mayor que el agua que era mucho más abundante y de mucha mayor utilidad? Surgió allí el concepto de utilidad marginal y se llegó a la conclusión que no es lo mismo analizar unidades por separado que stocks. Ludwig von Mises fue muy claro en este punto en su tratado de economía La Acción Humana:

El aprecio de las existencias totales de dos cosas puede diferir de la valoración de algunas de sus porciones. Un hombre aislado que posea siete vacas y siete caballos puede valorar en más un caballo que una vaca; es decir, que, puesto a optar, preferirá entregar una vaca antes que un caballo. Sin embargo, ese mismo individuo, ante la alternativa de elegir entre todos sus caballos y todas sus vacas, puede preferir quedarse con las vacas y prescindir de los caballos. ${ }^{24}$

Que el valor sea subjetivo es lo que permite que exista intercambio. Y si el intercambio es libre, las personas que participan del mismo, lo realizan porque esperan beneficiarse ya que de lo contrario no realizarían el intercambio. ${ }^{25}$ Los individuos tendrán

24 Von Mises (1949), p. 147.

25 Por supuesto puede ocurrir que luego de realizar el intercambio no quede satisfecha la persona. En ese caso habría incurrido en un error, pero al momento de realizar el intercambio esperaba beneficiarse por más que ex post no se haya beneficiado. 
sus diversas escalas de valoraciones facilitando la posibilidad de intercambio. En el libre intercambio las personas persiguen su interés personal. Y eso no está mal ya que como mencionaba Adam Smith, el bienestar individual terminará por generar el bienestar general. Esto es lo que predicaba con su famosa «mano invisible». Esta mano invisible es el equivalente a lo que Hayek denominó «orden espontáneo». Dicho orden es tan complejo que es muy difícil que pueda ser dirigido por una mente o por un gobernante de turno.

Ahora bien, para que haya libre intercambio es necesario que existan los derechos de propiedad. Nadie puede intercambiar lo que no es suyo. Por eso es importante la propiedad privada, para que cada uno tenga bienes de su posesión para intercambiar. El derecho de propiedad no puede ser ignorado ya que si se cree en el libre intercambio, nadie se esforzaría por producir si luego lo que él produce no podrá ser de su propiedad. ${ }^{26} \mathrm{~A}$ su vez esto le limita las posibilidades de intercambio con otros agentes económicos. Otra gran ventaja de la propiedad privada es que el individuo se verá tentado a vigilar de sus bienes con un cuidado especial ya que los obtuvo con el sudor de su frente. Si no existiera la propiedad privada esta motivación de cuidado se perdería deteriorando así además la calidad de los bienes.

Con respecto a la información, ocurren dos cosas. Primero que la racionalidad del hombre es limitada y segundo, como señala el economista Friedrich von Hayek, la información se encuentra dispersa. Este punto ya se ha mencionado más arriba pero vale la pena repetirlo ya que si el supuesto de conocimiento se relaja, muchas teorías económicas quedan en tela de juicio. Como ya se ha mencionado, si la información fuera perfecta se estaría en condiciones de realizar ejercicios de maximización y minimización para alcanzar la eficiencia económica. Lamentablemente el supuesto es irreal. No es por casualidad que más tarde comenzaron a aparecer conceptos como «costos de aprendizaje» $\mathrm{o}$ «learning by doing» que buscaban solucionar el problema de la información, es decir, se admitía que había un costo por adquirir la información.

${ }^{26}$ En caso de que no exista la propiedad privada. 
Pero si no se puede conocer toda la información, ¿cómo se opera en la economía? ¿Existe alguna guía?

La manera de interpretar la información es mediante el mecanismo de precios que guiará a los consumidores y productores para que realicen sus operaciones. Los precios tienen la increíble virtud de transmitir la información que no podemos conocer con un simple número. Guía tanto a oferentes como consumidores. El rol del precio en la Escuela Austriaca de Economía fue de tal importancia que Hayek hablaba de «El milagro del sistema de precios». Hayek señala:

En resumen, por medio de una especie de símbolo, sólo la información más esencial es comunicada, y es comunicada sólo a aquellos que les concierne. ${ }^{27}$

Continúa señalando Hayek más abajo:

Lo maravilloso es que en un caso como el de la escasez de una materia prima, sin que se dicte ninguna orden ni que la causa sea de ello sea conocida más que, tal vez, por una decena de personas, ocurre que millones de personas, cuya identidad no podría ser determinada con meses de investigación, reduzca el uso de la materia prima o sus productos; es decir, de hecho sucede que se mueven en la dirección correcta. Esta es ya una maravilla. ${ }^{28}$

El punto de Hayek es muy claro. Si poseo los recursos A, B y $C$ para producir un bien $X$ o un bien $Y$, si el precio de $X$ es mayor al precio de $Y$ (o si crece en mayor proporción que el que lo hace el de Y) me está dando la pauta de que ese mercado es más rentable y que la gente se encuentra demandando el bien $X$.

Como no podemos conocer toda la información, los precios son la guía para tomar nuestras decisiones. Es esto lo que se denomina proceso de mercado. Este proceso es un proceso de descubrimiento en donde el empresario es el actor principal. Al no ser perfecta la información entra en juego la incertidumbre. ${ }^{29}$ Es el

27 Hayek, op. cit., p. 165.

${ }^{28}$ Hayek, op. cit., p. 166.

${ }^{29}$ Esta variable no existe en la economía neo-clásica debido a que suponen que la información es perfecta. 
empresario que mediante el alertness ${ }^{30}$ descubre posibilidades de beneficios que creen valor. Si el empresario acierta genera un beneficio, si se equivoca incurrirá en pérdidas. El sistema de competencia se encargará de que los empresarios que tengan más desarrollado su alertness satisfagan las necesidades de los consumidores.

Por esta razón el mercado no se encuentra en equilibrio, sino en desequilibrio. La Escuela Austriaca de Economía analiza el proceso de mercado para acercarse al equilibrio, un equilibrio que nunca se alcanzará debido a que las circunstancias de tiempo y lugar varían día a día. La eficiencia económica es algo a los que el mercado se aproxima pero nunca llega. Cada oportunidad de beneficio que no se descubrió aún por el empresario es un estado de desequilibrio y a su vez es, como señala Israel Kirzner, una oportunidad explotable de ganancia.

Los empresarios se verán incentivados por los beneficios que puedan obtener, buscarán oportunidades para explotar ya sea nichos no desarrollados, posibilidades de arbitraje, innovación, etc. El punto es que cada una de sus acciones nos acerca a la eficiencia económica y al equilibrio. No podemos saber cuál es el punto de la eficiencia económica pero si podemos saber que el proceso de mercado nos acerca al mismo. Con lo cual, las características mencionadas de la Escuela Austriaca de Economía se vuelven importantes para alcanzar la eficiencia económica.

Para mostrar la importancia que posee el sistema de precios, sin intervención, para lograr la eficiencia económica volveremos al gráfico que se venía analizando, pero esta vez comparándolo con un gráfico adicional (Gráfico 8).

El gráfico siguiente muestra el punto de equilibrio entre la cantidad de lanchas y su precio cuando no hay un gobierno que intervenga. Como se puede apreciar, en ambos gráficos el eje de las $\mathrm{X}$ representa la cantidad de lanchas, lo que nos permite trasladar el punto de equilibrio del gráfico superior al punto de la FPP que realmente es eficiente, económicamente hablando.

30 Este término es introducido por Israel M. Kirzner y lo que implica es el estado de alerta de los empresarios para descubrir nuevas oportunidades de beneficios que no han sido explotadas. 
GRÁFICO 8

LA EFICIENCIA ECONÓMICA Y LA ESCUELA AUSTRIACA

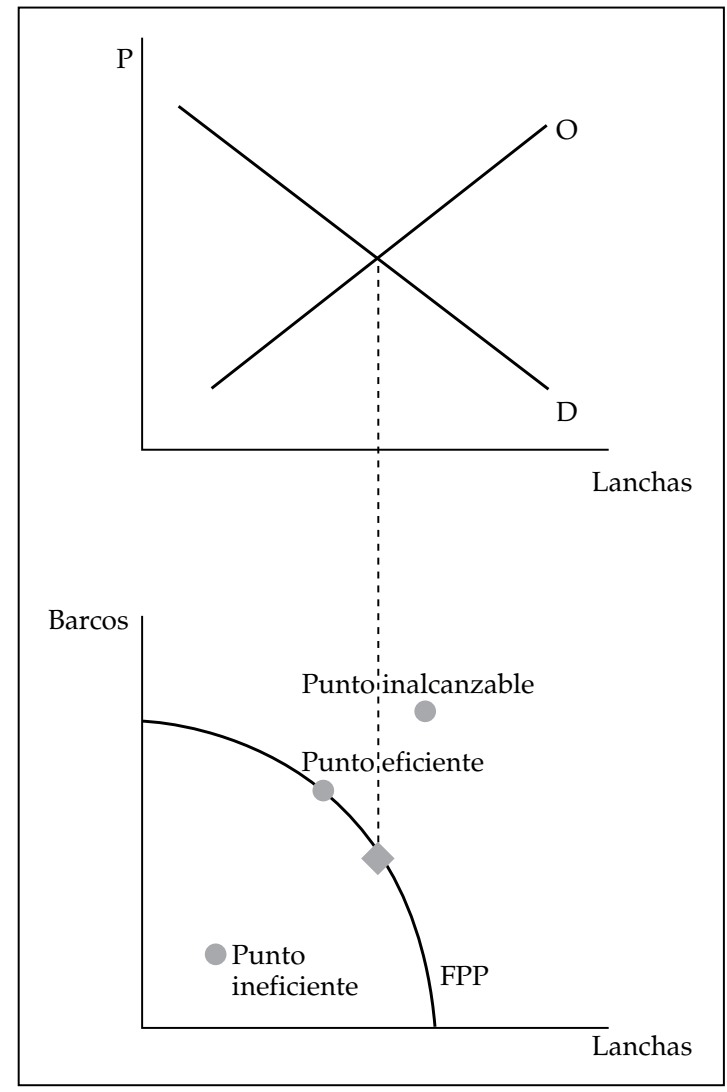

Finalmente, podemos ver que el punto inicial de producción (redondel) señalado sobre la FPP no brindaba una eficiencia económica, a pesar de encontrarse situada sobre la FPP. El que brinda la eficiencia económica es el cuadrado con forma de diamante. Mientras que las recetas keynesianas nos mantienen iterando en ida y vuelta desde un punto de la FPP a un punto ineficiente; el libre mercado nos orienta mediante el mecanismo de los precios, hacia el punto óptimo en términos técnicos y económicos. Es importante repetir para una mayor claridad, la eficiencia económica no se alcanzará en su totalidad debido a los constantes cambios que 
sufre la economía pero es el mecanismo que más nos acerca a dicho punto.

Por último, Jesús Huerta de Soto, realiza un comentario enriquecedor sobre este debate que se analizará a continuación.

\section{$\mathrm{V}$}

\section{LA TEORÍA DE LA EFICIENCIA DINÁMICA}

Jesús Huerta de Soto, en su artículo La teoría de la eficiencia dinámica crítica también el punto de la eficiencia técnica. Pero además, genera un enfoque más que interesante. El autor sostiene que los economistas se centran en discutir cómo llegar a la FPP y a la eficiencia económica cuando tal vez el debate debería girar en torno al proceso de descubrimiento. ${ }^{31}$ Señala Huerta de Soto:

Se trata, en suma, de aumentar los bienes por vía de la creatividad empresarial, es decir, del comercio y la especulación, más que evitando el despilfarro de los recursos que ya se poseen. ${ }^{32}$

En este sentido entraríamos en un proceso dinámico ya que la creatividad de los empresarios y sus inversiones estarían generando cambios al igual que la escala valorativa de los consumidores. De allí el concepto de eficiencia dinámica. El punto señalado por Huerta de Soto implicaría lo siguiente en términos gráficos (Gráfico 9).

Como se puede apreciar, lo importante es estar constantemente expandiendo la FPP para lograr desarrollo económico. En su trabajo, Huerta de Soto hace un recorrido de varios autores hasta llegar al concepto de eficiencia dinámica, pero es posible darle un cierre a la idea con la siguiente cita:

... lo verdaderamente relevante, más que llevar el sistema hacia la frontera de posibilidades máximas de producción (considerando «dada» la correspondiente curva), consiste en aplicar sistemáticamente el criterio de eficiencia dinámica, que es aquel que

31 En concordancia con la Escuela Austriaca de Economía.

32 Huerta de Soto (2004), p. 15. 
GRÁFICO 9

EFICIENCIA DINÁMICA

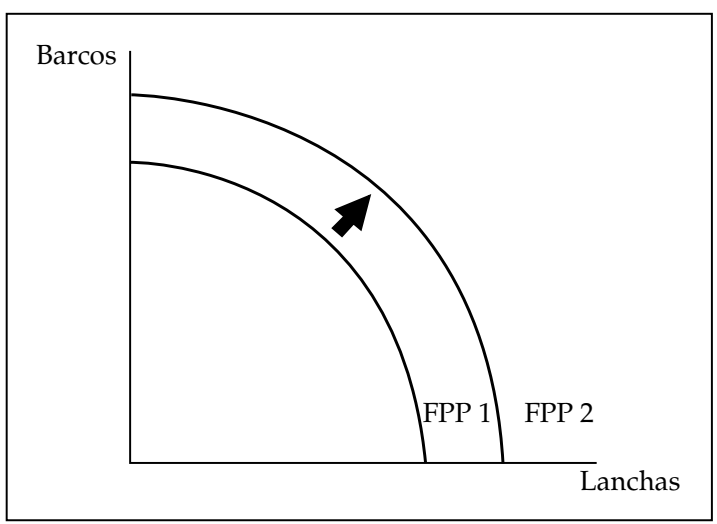

se fija en la capacidad del sistema para «mover» continuamente hacia la derecha la curva de posibilidades máximas de producción. ${ }^{33}$

Teniendo en cuenta el apartado anterior y éste podríamos resumir que el mecanismo de precios es el que se ocupa de acercarnos a la eficiencia económica mientras que la teoría de la eficiencia dinámica, por medio del empresario, se concentra en expandir la FPP.

\section{VI \\ CONCLUSIÓN}

En conclusión, no se debería pretender conocer más de lo que nuestras capacidades nos permiten. Además, como ya se mencionó, la información se encuentra dispersa y con el efecto de la globalización se dispersó aún más. Resultaría imposible que una persona cuente con toda la información para tomar las decisiones correctas teniendo en cuenta las valoraciones subjetivas de

33 Huerta de Soto, op. cit., pp. 25-26. 
los individuos. Por ejemplo, un funcionario público puede opinar que en una determinada provincia hace falta desarrollar la industria del calzado mientras que otro funcionario puede opinar que la industria que hace falta desarrollar es la del cobre. Lo más probable es que ambos estén equivocados. Ambos desperdician recursos intentando dirigirse hacia un punto sobre la frontera de posibilidades de producción ineficiente en términos económicos. El rol de los precios a través del libre mercado nos brindará la información necesaria para poder optar por la decisión correcta y así lograr la eficiencia económica. Cualquier otro punto sobre la FPP hubiera terminado por desperdiciar recursos productivos consecuencia de una mala lectura del gobernante de turno.

Además, mientras que por un lado los precios nos aproximan a la eficiencia económica, por el otro, los empresarios con su alertness e innovaciones desarrollan la eficiencia dinámica expandiendo la FPP hacia la derecha. La economía convencional podrá ser capaz de mostrar un punto en donde la FPP realiza tangencia con la curva de indiferencia y así logra una eficiencia económica, pero sólo lo alcanza con supuestos discutibles. Lamentablemente al haber información imperfecta el mecanismo que más se aproxima hacia la eficiencia económica es el libre mercado basado en la teoría que tanto hincapié realizó la Escuela Austriaca de Economía. Tal vez sea hora de que los economistas modifiquen su perspectiva a la hora de encarar los problemas económicos. Hubo muchos economistas que realizaron observaciones acerca de la subjetividad del valor y su importancia y de no basarse en supuestos alejados de la realidad. Se ha observado que de lo contrario se puede llegar a conclusiones erróneas. En palabras de James M. Buchanan:

Deseo que los economistas modifiquen sus procesos de pensamiento, que contemplen los mismos fenómenos desde «otra ventana», por utilizar la apropiada metáfora de Nietzsche. Deseo que se concentre en el intercambio más que en la elección. ${ }^{34}$

34 Buchanan (1964), p. 124. 


\section{REFERENCIAS BIBLIOGRÁFICAS}

Benegas, A. (1972): Fundamentos de análisis económico, 12. ed. Buenos Aires: ISA Ediciones. 2011.

BUCHANAN, J.M. (1964): «¿Qué deberían hacer los economistas?». Libertas, N.o 1, Octubre 1984, pp. 117-134.

CACHANOSKY, J.C. (Septiembre 1994): «Certidumbre, incertidumbre y eficiencia económica.» Laissez-Faire, N.․ 1, pp. 30-42.

HAYEK, F.A. von (1945): «El uso del conocimiento en la sociedad». Estudios Públicos, N. ${ }^{12}$, 1983, pp. 157-169.

- (1948): «El significado de la competencia». Libertas, N. 13 , Octubre 1990, pp. 263-280.

HazlitT, H. (1946): La economía en una lección, 2. a ed. Madrid: Unión Editorial. 1981.

Huerta De SOTO, J. (2004): «La teoría de la eficiencia dinámica».

Procesos de Mercado: Revista Europea de Economía Política, Vol. I, N.․ 1, pp. 11-71.

IrigoIN, A.M. (Octubre 1998): «Desarrollo económico y función empresarial». Libertas, ‥ 9, pp. 183-206.

KEYNES, J.M. (1930): «The great slump of 1930». http:/ / gutenberg. ca/ebooks / keynes-slump/keynes-slump-00-h.html

KIRZNER, I.M. (Enero 1965): «What economist do». Southern Economic Journal, Vol. 31, N. 3, pp. 257-261.

- (1986): «El empresario». Libertas, ‥ 29, 1998, pp. 49-100.

- (1990): «El significado del proceso de mercado». Libertas, N.o 27, Octubre 1997, pp. 121-140.

KraUSE, M.E. (2011): Economía, instituciones y políticas públicas, 1. a ed. Buenos Aires: La Ley.

Ledesma, J. (2003): Economía. Teoría y política, 1. a ed. Buenos Aires: Pearson.

Mises, L. von (1927): Liberalism, 4. a ed. Indianapolis: Liberty Fund.

- (1949): La acción humana. Tratado de economía. 7.. ed. Madrid: Unión Editorial.

Morgenstern, O. (1972): «Thirteen critical points in contemporary economic theory: an interpretation». Journal of Economic Literature. American Economic Association. Vol. 10 (4), pp. 1163-1189. 
Nicholson, W. (2005): Teoría microeconómica. Principios básicos y

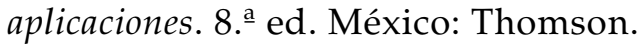

RAvier, A. (2010): En busca del pleno empleo. Estudios de macroeconomía austriaca y economía comparada. 1. ed. Madrid: Unión Editorial.

READ, L. (1958): «I, pencil: My family tree». Essays of Liberty. Foundation for Economic Education. Vol. VI, pp. 371-379.

Rothbard, M.N. (1962): Man, economy and state. 1. a ed. Los Angeles: Nash Publishing.

VARIAN, H.R. (1999): Microeconomía Intermedia. 5. a ed. Barcelona: Antoni Bosch editor. 